\title{
Histopathological variant types diagnosed in prostate surgery
}

\author{
Semih Aktaş ${ }^{1}$, Hikmet Köseoğlu², Uğur Yücetaş ${ }^{3}$, Sevim Baykal Koca ${ }^{4}$
}

Cite this article: Akta S, Köseoğlu H, Yücetaş U, Koca SB: Histopathological variant types diagnosed in prostate surgery. Ann Urol Oncol 2020; 3(1): 47-53. https://doi.org/10.32948/ auo.2020.12.30

\begin{abstract}
Rare pathological variants may only be diagnosed after the surgical treatments applied. We aimed to determine the rare variant pathologies and their frequencies diagnosed in prostate surgeries performed in routine patient care. We retrospectively analyzed hospital pathology records for all prostate surgeries performed namely TUR-P, open prostatectomy, and radical prostatectomy between October 2014 and October 2019. A total of 1345 patients' clinical data together with relevant prostate surgery pathologic diagnoses were evaluated from the database. Their mean age was $67.63 \pm 8.09$. The most common comorbid diseases were hypertension (46\%), diabetes mellitus (21\%) and cerebrovascular disease $(23 \%)$. Surgeries indicated and performed for prostate diseases were TUR-P (72\%), open prostatectomy $(9.7 \%)$ and radical prostatectomy $(18.4 \%)$. The respective median PSA values for above mentioned surgery groups were $3.21,7.34$ and $8.13 \mathrm{ng} / \mathrm{ml}$. Ninety-three patients $(6.9 \%)$ had variant pathology associated with their primary pathologies including either BPH or prostate adenocarcinoma. $8.6 \%$ of patients with variant pathology had more than one variant types. Histopathological variant types generally considered rare can be seen up to $20 \%$ in oncological cases according to our database and their clinical importance and treatment differs for each variant type.
\end{abstract}

Key words Prostate, histopathology, variant, carcinoma, hyperplasia

\footnotetext{
1. Health Sciences University Istanbul Training and Research Hospital, Urology Clinics, Istanbul.

2. FEBU, FECSM, Health Sciences University Istanbul Training and Research Hospital, Urology Clinics, Istanbul.

3. Health Sciences University Istanbul Training and Research Hospital, Urology Clinics, Istanbul.

4. Health Sciences University Istanbul Training and Research Hospital, Pathlogy Clinics, Istanbul.

Correspondence: Hikmet Köseoğlu, MD, PhD, Associate Professor, FEBU, FECSM, FACS (Department of Urology, University of Health Sciences, Istanbul Training and Research Hospital, Kasap Ilyas Mah, Org. Abdurrahman Nafiz Gurman Cad, 34098 Fatih/İstanbul TR; Email: hikmet.koseoglu@ gmail.com).
} 


\section{Introduction}

The prostate gland is a reproductive organ, which has different embryological zones, originating from different structures, namely the central zone form Wolf channel and both the transitional and peripheral zone from the urogenital sinus. The prostate is histologically composed of glandular epithelium and fibromuscular stroma $[1,2]$. Benign and malignant histopathological types of prostate lesions were lastly updated by World Health Organizaion (WHO) in 2016 [3]. Acinar prostatic adenocarcinoma variants according to WHO are entitled as pseudohyperplastic, atrophic, foamy, microcystic, mucinous, signet ring-like cell, pleomorphic giant cell variant and sarcomatoid. Non-acinar prostatic adenocarcinoma variants include intra-ductal carcinoma, ductal carcinoma, small cell carcinoma, urothelial carcinoma, and neuroendocrine tumors etc. [3]. Benign prostatic hyperplasia $(\mathrm{BPH})$ is the most common cause of surgical intervention in benign forms of the prostate and adenocarcinomas are the most common in malignant phenotype. Rare pathological variants may only be diagnosed after the application of various surgical treatments.

Especially in biopsy pathologies, there may be difficulties in diagnosing variant types and may be confused with standard adenocarcinoma. Else, the surgical treatment and follow-up protocol applied according to the histopathological types may differ. Although well associated variant pathologies differ clinically in progression, survival, treatment, and follow-up disease compared to common acinar prostatic adenocarcinomas $[4,5]$.
The present study aimed to determine retrospectively associated variant pathologies and their frequencies in pathological specimens of the prostate surgeries performed in routine patient care.

\section{Material \& methods}

All patients' hospital data record was retrospectively searched for the patients operated with the operation codes "TUR-P, open prostatectomy or radical prostatectomy" during the last 5 years from October 2014 to October 2019. The patients with unclear pathology reports or missing clinical data were excluded. For the evaluation, a total of 1345 patients were included after the exclusion criteria.

Relevant clinical data together with operations' details and pathology reports were noted. All reported pathological diagnoses of relevant prostate surgeries including variant pathologies were re-checked by the pathologist for their validity together with their primary pathologies and with all relevant patients' clinical data.

Tissue samples of pathological specimens $(4-6 \mu \mathrm{m})$ were fixed in formalin, parafin-embedded onto glass slides and stained with hematoxylin and eosin for histological evaluation. The slides were then examined under a light microscope (BW51; Olympus Corporation Tokyo, Japan). Immunohistochemical studies were performed for $\mathrm{p} 63,34 \beta \mathrm{E} 12, \alpha$-methylacyl-coenzyme A racemase (AMACR), synaptophysin, chromogranine, caldesmon, alfasmooth-muscle-actin. Immunohistochemistry was conducted using the Benchmark XT staining system (Ventana Medical

Table 1. Variant types and frequencies according to surgery types.

\begin{tabular}{lll}
\hline Common variants & TUR-P/Open Prostatectomy N=1098 & Radical Prostatectomy N=247 \\
\hline Intraductal adenocarcinoma & $13(1.2 \%)$ & $50(20.2 \%)$ \\
Ductal adenocarcinoma & $2(0.2 \%)$ & $4(1.6 \%)$ \\
Foamy gland type adenocarcinoma & - & $8(3.2 \%)$ \\
Squamous metaplasia* & $4(0.4 \%)$ & - \\
Urothelial carcinoma* & $2(0.2 \%)$ & \\
Polipoid urethritis & $2(0.2 \%)$ & - \\
Leiomyoma & $2(0.2 \%)$ & $1(0.4 \%)$ \\
Neuroendocrine tumor of prostate & - &
\end{tabular}

Mucinous (colloid) variant of acinar

adenocarcinoma

Signet ring-like variant of acinar

adenocarcinoma

Atrophic variant adenocarcinoma

Small cell carcinoma*

Other associated variants with detected rate lesser than $\% 1$

Solitary fibrous tumor

Inverted papilloma

Granuloma*

*: Relevant pathology slides are shown in Figure 1,2,3 except non-prostate specific ones. 

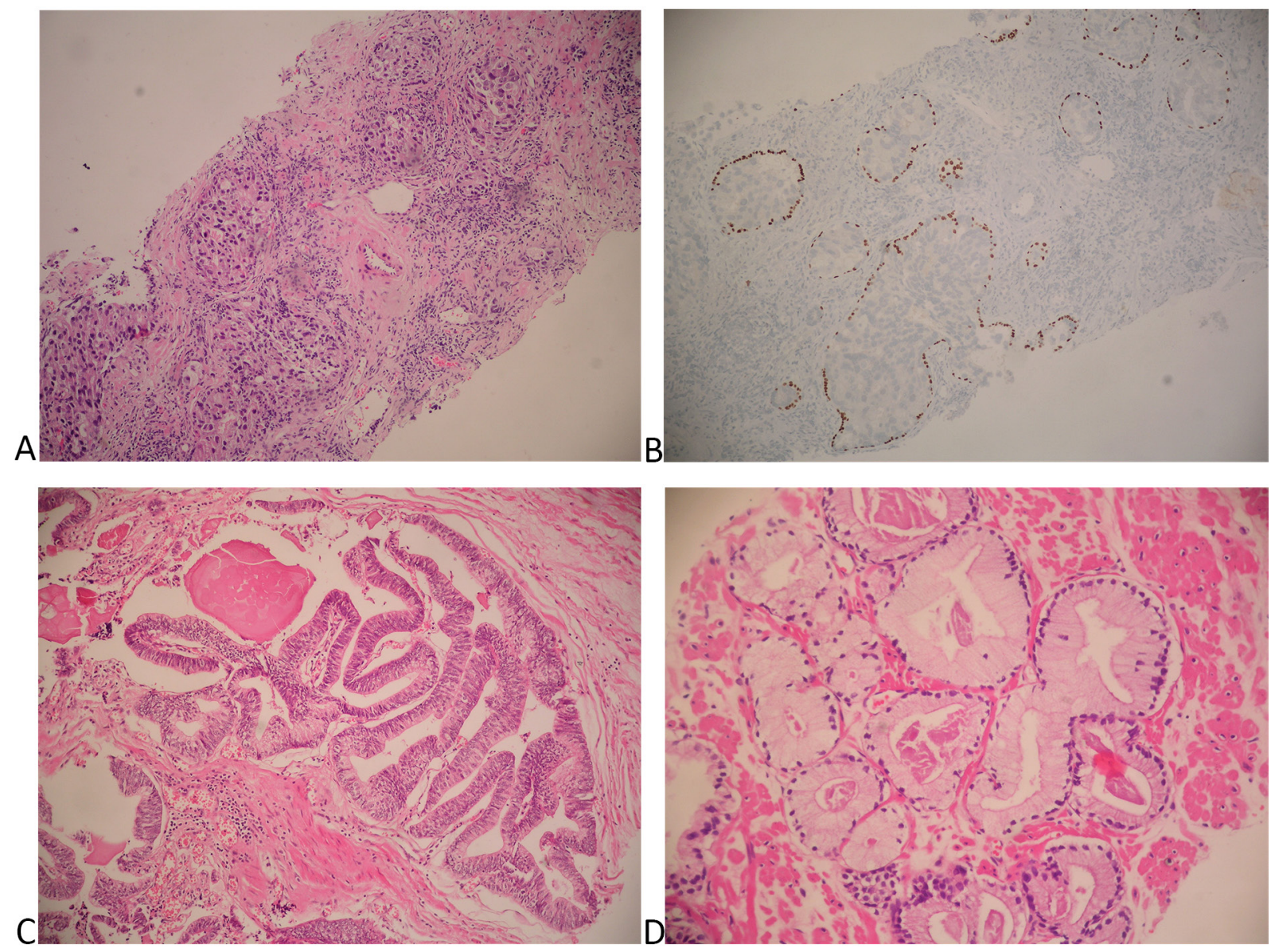

Figure 1. Pathological slides of common histopathological variants (rate above 1\%) within our series. (A) Intraductal adenocarcinoma there are solid and cribriform pattern glands with marked nuclear pleomorphism (H\&E, x200); (B) Intraductal adenocarcinoma; Immunohistocehical P63 stain highlights the presence of basal cells at the periphery of the lesion (x200); (C) Ductal adenocarcinoma with papillary growth and pseudostratified columnar epithelium (H\&E, x100); (D) Foamy gland variant of adenocarcinoma with xanthomatous cytoplasm and pycnotic nuclei (H\&Ex200).

Systems, Inc., Tucson, AZ, USA) and antibodies against 34ßE12 (monoclonal mouse anti-human; cat. no. CM 127 AC; 1:50; Biocare Medical Inc., Concord, CA, USA), AMACR (monoclonal rabbit anti-human; cat. no. 504S-14; 1:100; Cell Marque Corporation, Rocklin, CA, USA) and p63 (monoclonal mouse anti-human; cat. no. VP 163 GG25; 1:200; Biocare Medical Inc.), synaptophysin (antibody polyclonal, dilution: 1:500, CellMarque Sigma- Aldrich Co, Rocklin, CA, USA) and chromogranin A (antibody LK2H10, dilution: 1:500, CellMarque Sigma-Aldrich Co, Rocklin, CA, USA)

Briefly, the tissue sections were deparafinized with EZ Prep solution (Ventana Medical Systems, Inc.) at $75^{\circ} \mathrm{C}$, pretreated with cell conditioning 1 (CC1) solution (Ventana Medical Systems, Inc.) for antigen retrieval at $95^{\circ} \mathrm{C}$, and incubated with hydrogen peroxide (Ventana Medical Systems, Inc.) for 4 min to block endog- enous peroxidase activity. The sections were then incubated with the AMACR, p63 and 34ßE12 primary antibodies for $32 \mathrm{~min}$ at 37 ${ }^{\circ} \mathrm{C}$. Next, the sections were blocked using the Endogenous Biotin Blocking Kit (Ventana Medical Systems, Inc.) followed by incubation with a streptavidin-horseradish peroxiade-conjugated secondary antibody (monoclonal goat anti-rat; cat. no. 760-500; 1:200; Ventana Medical Systems, Inc.) for $8 \mathrm{~min}$ at $37^{\circ} \mathrm{C}$. The immunolocalized AMACR, p63 and 34ßE12 were visualized using a copper-enhanced DAB reaction. The slides were counterstained with hematoxylin II (Ventana Medical Systems, Inc.) for 4 min and Bluing Reagent (Ventana Medical Systems, Inc.) for 4 min and coverslips were applied using an automated coverslipper (TissueTek Film Automated Coverslipper; Sakura Finetek Japan Co., Ltd., Tokyo, Japan). Cytoplasmic staining for AMACR and 34ßE12, and nuclear staining for $\mathrm{p} 63$ were considered positive.

Among all patients' pathology slides which were already stained necessarily and stored in the pathology archive room, best preserved and demonstrative ones were chosen to be presented.

\section{Results}

The average age of 1345 patients included in the study was $67.63 \pm 8.09$. The most common comorbid disease noted were hypertension (46\%), diabetes mellitus (21\%) and cerebrovascular disease $(23 \%)$. Surgeries indicated and performed for prostate diseases were TUR-P (72\%), open prostatectomy (9.7\%) and radical prostatectomy (18.4\%). The respective median PSA values for above mentioned surgery groups were $3.21,7.34$ and $8.13 \mathrm{ng} /$ $\mathrm{ml}$.

Ninety-three patients (6.9\%) had variant pathology associated with their primary pathologies including either BPH or prostate adenocarcinoma. $8.6 \%$ of patients with variant pathology had more than one variant types. Variant types and frequencies 

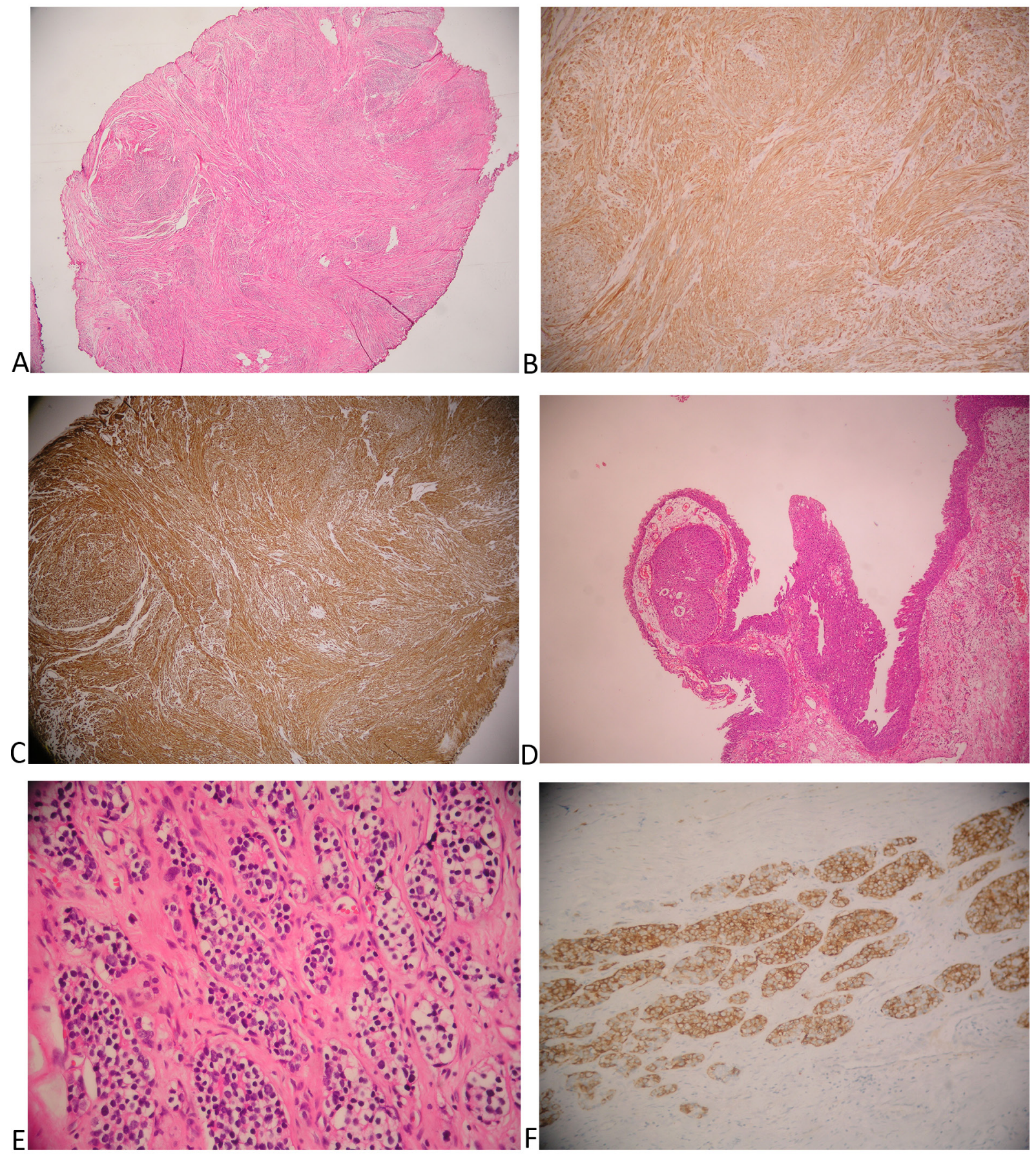

Figure 2. Pathological slides of common histopathological variants (rate between $0.1 \%$ and $1 \%$ ) within our series. (A) Leiomyoma of prostate there are smooth muscle cells with bland cytology form fascicules (H\&E, x40); (B) Caldesmon immunohistochemical stain showing strong cytoplasmic reactivity in tumor cells (x40); (C) Muscle specific actin immunohistochemical stain showing strong cytoplasmic reactivity in tumor cells (x100); (D) Polypoid urethritis (H\&E, x100); (E) Neuroendocrine tumor of the prostate with nests of cells with uniform round nuclei with 'salt and pepper" chromatin (H\&E, x200); (F) Synaptophysin immunohistochemical stain showing strong positive reaction in tumor cells (x200).

according to surgery types are shown in the Table 1 . The associated variant pathologies were intraductal adenocarcinoma, ductal adenocarcinoma, foamy gland type adenocarcinoma, squamous metaplasia, urothelial carcinoma, polypoid urethritis, leiomyoma, neuroendocrine tumor of prostate, mucinous (colloid) variant of acinar adenocarcinoma, signet ring-like variant of acinar adenocarcinoma, atrophic variant adenocarcinoma, small cell carcinoma, solitary fibrous tumor, inverted papilloma and granuloma in order of their detected rates. The relevant pathology sample slides are shown in Figures 1-3 except non-prostate specific ones, namely squamous metaplasia, urothelial carcinoma, small cell carcinoma, granuloma .

\section{Discussion}

The prostate remains one of the major surgical organs for men 

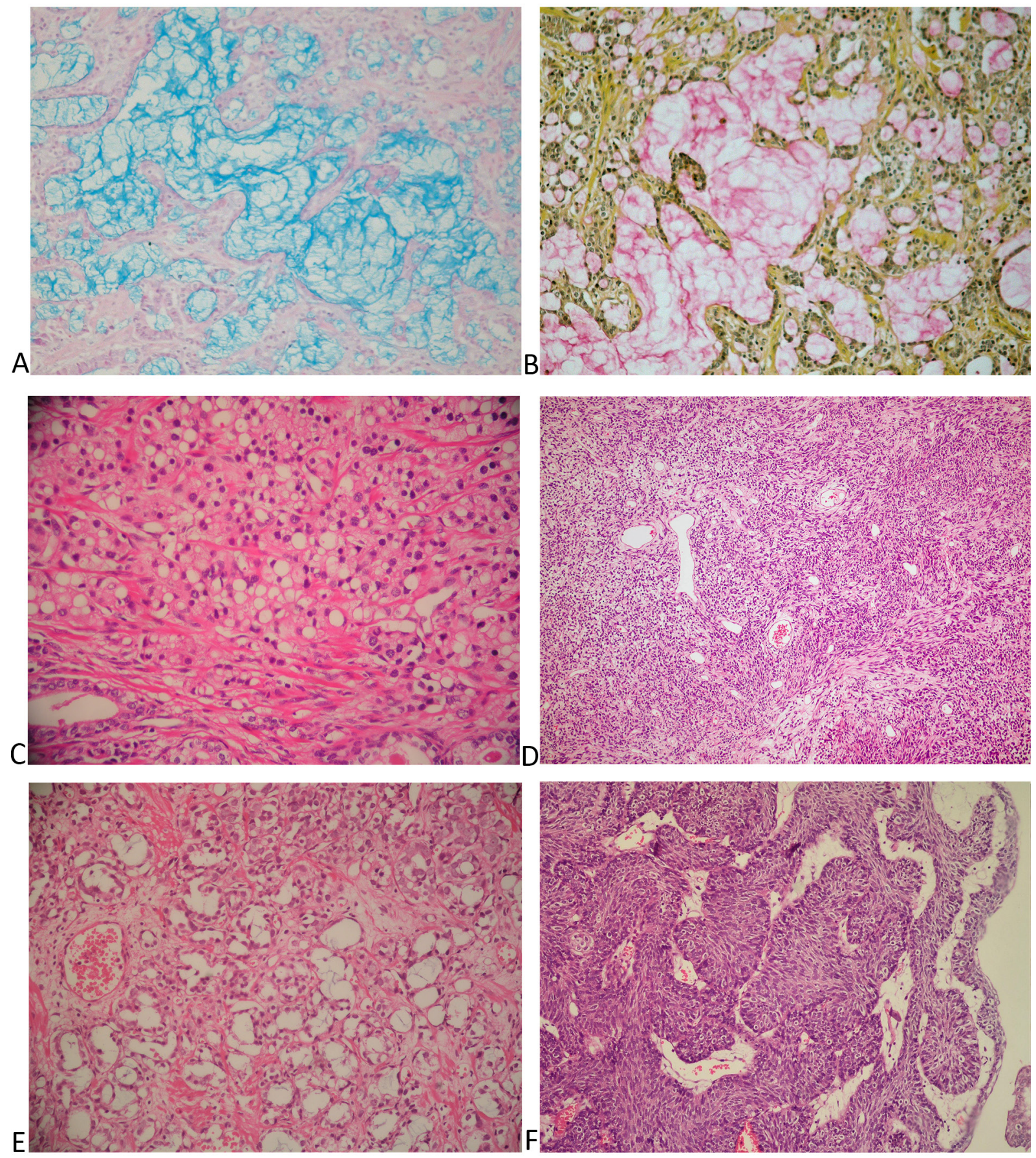

Figure 3. Pathological slides of common histopathological variants (rate less than $0.1 \%$ ) within our series. (A) Mucinous (colloid) variant of acinar adenocarcinoma with alcian blue positive mucin lakes (Alcian blue, x100); (B) Mucinous (colloid) variant of acinar adenocarcinoma with mucicarmine positive mucin lakes (Mucicarmine, x100); (C) Signet ring-like variant of acinar adenocarcinoma with nuclear displacement and cytoplasmic vacuole formation. In the absence of mucin production, the term signet ring-like adenocarcinoma is used (H\&E, $x 200)$; (D) Solitary fibrous tumor (H\&E, x200); (E) Atrophic variant of acinar adenocarcinoma. Atrophic-appearing malignant glands are admixed with prostatic carcinoma of the usual type with moderate amounts of amphophilic cytoplasm (H\&E, x200); (F) Inverted papilloma of the prostatic urethra there are downward growing anastomosing trabeculae of urothelium with peripheral palisading and central streaming (H\&Ex200).

over fifty for either BPH related LUTS and/or malignancy [6]. However, evaluation of preoperative biopsies or surgical excision specimens' pathologies may have challenges due to the confusable variant pathologies [7]. These variants have been defined by WHO as pseudohyperplastic, atrophic, foamy, microcystic, mucinous, pleomorphic giant cell variant and sarcomatoid [3]. The importance of variant pathologies lies in the fact that presence of variant type might have effect on the progression, survival, treatment, and follow-up of the disease compared to acinar prostatic adenocarcinomas $[4,5]$. Recent publications suggest that the presence of variant types, especially in cases of castrationresistant prostate cancer is associated with increased biochemical recurrence and metastasis, androgen suppression and resistance to chemotherapy despite multimodal treatments [8]. 
In this present study the commonest variant type; IDC-P was detected in $20 \%$ in the radical prostatectomy specimens, which seems to be a rather important and notable rate. While IDC-P is generally seen with invasive carcinomas, it can also be found in isolated forms [9, 10]. IDC-P is associated with adverse clinicpathological parameters such as large tumor volume, high grade Gleason score, extra prostatic enlargement and seminal vesicle invasion [11]. Likewise, active surveillance is not recommended for patients with localized prostate cancer otherwise indicated [12]

While ductal adenocarcinoma of the prostate is usually associated with acinar adenocarcinomas in pathology preparations after radical prostatectomy, it can also be found in pure form. DAC was previously called endometrioid adenocarcinoma. Lee et al reported a DAC incidence of $0.4 \%$, while in the series of Bostwick et al. this was $0.8 \%$ detected $[13,14]$.

It has not been mentioned whether it is associated with pure DAC or acinar adenocarcinoma. 1051 radical prostatectomy preparations evaluated retrospectively in 2017 were divided into subgroups according to their evaluated morphological characteristics. DAC incidence was determined as $8.2 \%$ in total [15]. In our series, this rate was determined as $1.4 \%$. After radical prostatectomy, shorter biochemical recurrence rates, higher mortality, extraprostatic spread, seminal vesicle invasion, local and regional metastases and positive surgical margins are observed. DAC causes difficulties in its follow-up and treatment because it metastasizes to less common areas for prostate cancer such as the lung, brain, testicle and penis $[16,17]$. Therefore, it should be kept in mind that this variant type may be found in atypical localizations in follow-up and treatment modalities and should be kept in mind in early postoperative PSA recurrences.

Foamy gland prostate adenocarcinoma is classified as intermediate carcinoma according to 2016 who. Although generally characterized by Gleason $3+3$, Gleason can be found in $3+4$ or $4+3$ patterns. When pathology specimens of 477 patients who had radical prostatectomies were evaluated, foamy was found at a rate of $69(14.5 \%)$. Gleason 7 pattern was detected in $63 \%$ of the patients with foamy [18]. In another study, foamy detected after biopsy and TUR-P in 2009 was also between the Gleason score (68). Therefore, it is one of the variants to be considered [19].

When 2648 autopsy patients were evaluated in the 1980s for prostate adenocarcinoma, which is one of the variants with a very poor prognosis, prostate cancer was detected in 69 patients and 8 $(11.5 \%)$ of them had cancer with neuroendocrine differentiation [20]. However, although its incidence is not clearly known in recent studies, prostate carcinoma with such a high rate of neuroendocrine differentiation is not seen.

Mucinous adenocarcinoma of the prostate is one of the rare variants of the prostate and its incidence is around $0.2 \%$. In a cohort study of men under 50 years of age in 2017, this rate was found to be $6 \%$ [21]. This means that some variant types can be seen at much higher rates than expected in different age groups and different societies. For these reasons, it should be known that there are differences in the progression of each variant type while waiting for follow-up or treatment regimens.

Solitary fibrous tumor is one of the rare mesenchymal tumors of the prostate. Approximately 20 cases have been reported in the literature. While they show benign features around $80 \%$, they may show malignant character in $20 \%$ and relapse in the early period [22]. Though MRI may be helpful in diagnosis and staging, the definitive diagnosis of solitary fibrous tumor is made by immunohistochemical findings and histopathological features [23]. Its prognosis mainly depends on complete removal of the existing tumor after surgery and negative surgical margins because early local recurrences are common in those with an aggressive course [22].

Small cell prostate carcinoma of the prostate is a rare variant.
Causes lower urinary symptoms. It does not cause PSA elevation, but it may give signs of rectal touch. These tumors generally show an aggressive course. Sometimes, pathology preparations can be misinterpreted and evaluated as ISUP 4-5. Multimodal therapy used in prostate adenocarcinoma is applied to the patient. Small cell prostate carcinoma of the prostate is resistant to chemotherapy and hormonotherapy used in prostate adenocarcinoma. Therefore, the chemotherapy protocol is platinum-based agents and etoposide [24].

Inverted papilloma are rare benign tumors of the urethral system and can be confused with transitional cell carcinoma [25]. It can be observed in the ureter, bladder, bladder neck (most often), proximal and distal urethra. Though benign, recurrences can occur and be associated with transitional cell carcinoma. Therefore, it should be followed up cystoscopy.

The present study reports histopathological variant types associated with prostate diseases detected in specimens after surgeries, namely TUR-P, open prostatectomy, or radical prostatectomy. As a limitation the present study did not and is not able to address the correlation between the variants and the type of surgery performed.

\section{Conclusions}

Histopathological variant types generally considered rare can be seen up to $20 \%$ in oncological cases according to our database and their clinical importance and treatment differs for each variant type. The clinical significance and treatment of variants varies with each variant type. Therefore, correct determination of variant types will lead to changes in the follow-up and treatment schedule and will contribute more to prolonged biochemical recurrence time, progression and survival.

\section{Ethical policy}

Approval was taken from institutional ethical committee. The study was performed in accordance with the Declaration of Helsinki. Patients gave their informed consent for their participation.

\section{Author contributions}

SA and HK designed the study and drafted the manuscript. UY and SK prepared the figures and tables. All authors read and approved the final manuscript.

\section{Competing interests}

The authors declare no competing interests.

\section{References}

1. Aaron L, Franco OE, Hayward SW: Review of Prostate Anatomy and Embryology and the Etiology of Benign Prostatic Hyperplasia. Urol Clin North Am 2016, 43(3): 279-288.

2. Villers A, Steg A, Boccon-Gibod L: Anatomy of the prostate: review of the different models. Eur Urol 1991, 20(4): 261-268.

3. Humphrey PA, Moch H, Cubilla AL, Ulbright TM, Reuter VE: The 2016 WHO Classification of Tumours of the Urinary System and Male Genital Organs-Part B: Prostate and Bladder Tumours. Eur Urol 2016, 70(1): 106-119.

4. Humphrey PA: Histological variants of prostatic carcinoma and their significance. Histopathology 2012, 60(1): 59-74.

5. Mazzucchelli R, Lopez-Beltran A, Cheng L, Scarpelli M, Kirkali Z, Montironi R: Rare and unusual histological variants of prostatic carcinoma: clinical significance. BJU Int 2008, 102(10): 1369-1374. 
6. Lokeshwar SD, Harper BT, Webb E, Jordan A, Dykes TA, Neal DE $\mathrm{Jr}$, Terris MK, Klaassen Z: Epidemiology and treatment modalities for the management of benign prostatic hyperplasia. Transl Androl Urol 2019, 8(5): 529-539.

7. Watts K, Li J, Magi-Galluzzi C, Zhou M: Incidence and clinicopathological characteristics of intraductal carcinoma detected in prostate biopsies: a prospective cohort study. Histopathology 2013, 63(4): 574-579.

8. Porter LH, Lawrence MG, Ilic D, Clouston D, Bolton DM, Frydenberg M, Murphy DG, Pezaro C, Risbridger GP, Taylor RA: Systematic Review Links the Prevalence of Intraductal Carcinoma of the Prostate to Prostate Cancer Risk Categories. Eur Urol 2017, 72(4): 492-495.

9. Robinson BD, Epstein JI: Intraductal carcinoma of the prostate without invasive carcinoma on needle biopsy: emphasis on radical prostatectomy findings. J Urol 2010, 184(4): 1328-1333.

10. Watts K, Li J, Magi-Galluzzi C, Zhou M: Incidence and clinicopathological characteristics of intraductal carcinoma detected in prostate biopsies: a prospective cohort study. Histopathology. 2013, 63(4): 574-579.

11. Humphrey PA: Histopathology of Prostate Cancer. Cold Spring Harb Perspect Med 2017, 7(10): a030411.

12. Montironi R, Hammond EH, Lin DW, Gore JL, Srigley JR, Samaratunga H, Egevad L, Rubin MA, Nacey J, Klotz L, et al: Consensus statement with recommendations on active surveillance inclusion criteria and definition of progression in men with localized prostate cancer: the critical role of the pathologist. Virchows Arch 2014, 465(6): 623-628.

13. Bostwick DG, Kindrachuk RW, Rouse RV: Prostatic adenocarcinoma with endometrioid features. Clinical, pathologic, and ultrastructural findings. Am J Surg Pathol 1985, 9(8): 595-609.

14. Christensen WN, Steinberg G, Walsh PC, Epstein JI: Prostatic duct adenocarcinoma. Findings at radical prostatectomy. Cancer 1991, 67(8): 2118-2124.

15. Lee SS: Endometrioid adenocarcinoma of the prostate: a clinicopathologic and immunohistochemical study. J Surg Oncol 1994, 55(4): 235-238.

16. Seipel AH, Delahunt B, Samaratunga H, Egevad L: Ductal adenocarcinoma of the prostate: histogenesis, biology and clinicopathological features. Pathology 2016, 48(5): 398-405.

17. Seipel AH, Wiklund F, Wiklund NP, Egevad L: Histopathological features of ductal adenocarcinoma of the prostate in 1,051 radical prostatectomy specimens. Virchows Arch 2013, 462(4): 429-436.

18. Hudson J, Cao D, Vollmer R, Kibel AS, Grewal S, Humphrey PA: Foamy gland adenocarcinoma of the prostate: incidence, Gleason grade, and early clinical outcome. Hum Pathol 2012, 43(7): 974-979.

19. Zhao J, Epstein JI: High-grade foamy gland prostatic adenocarcinoma on biopsy or transurethral resection: a morphologic study of 55 cases. Am J Surg Pathol 2009, 33(4): 583-590.

20. Turbat-Herrera EA, Herrera GA, Gore I, Lott RL, Grizzle WE, Bonnin JM: Neuroendocrine differentiation in prostatic carcinomas. A retrospective autopsy study. Arch Pathol Lab Med 1988, 112(11): 1100-1105.

21. Prendeville S, Nesbitt ME, Evans AJ, Fleshner NE, van der Kwast TH: Variant Histology and Clinicopathological Features of Prostate Cancer in Men Younger than 50 Years Treated with Radical Prostatectomy. J Urol 2017, 198(1): 79-85.

22. Matos J, Paparo F, Calcagno T, Marinaro E, Introini C, Rollandi GA: Solitary Fibrous Tumor of the Prostate. Urology 2020, 141: e43-e44.

23. Sano K, Miyai K, Yoshida S: Small cell carcinoma of the prostate: a case report. Int J Urol 1997, 4(3): 321-323.

24. Lee SH, Mah SY, Chung BH: Incidentally discovered inverted papilloma of the urinary bladder in patients with lower urinary tract symptoms. J Endourol 2010, 24(2): 271-275.

25. Brown AL, Cohen RJ: Inverted papilloma of the urinary tract. BJU Int 2011, 107 Suppl 3: 24-26. 